\title{
Reimplante dentário após trauma de avulsão: Relato de caso clínico
}

\author{
Dental reimplantation after avulsion trauma: Clinical case report \\ Reimplantación dental tras traumatismo por avulsión: Informe de caso clínico
}

Recebido: 08/06/2021 | Revisado: 16/06/2021 | Aceito: 20/06/2021 | Publicado: 04/07/2021

Ernandi Ribeiro Cezar de Lima

ORCID: https://orcid.org/0000-0002-6783-3627 Centro Universitário Tiradentes, Brasil E-mail: ernandicezar@outlook.com

Gabriel Cortez da Silva Toledo

ORCID: https://orcid.org/0000-0002-0543-8840 Centro Universitário Tiradentes, Brasil E-mail: gabriel.cortez@souunit.com.br

Thaise Ravanny de Araújo Silva

ORCID: https://orcid.org/0000-0002-0167-140X Centro Universitário Tiradentes, Brasil E-mail: thaise.ravanny@souunit.com.br

Lucas Mariz de Menezes Torres

ORCID: https://orcid.org/0000-0001-7543-7715 Centro Universitário Tiradentes, Brasil E-mail: 1marizdemenezes@gmail.com

Rafaela Andrade de Vasconcelos

ORCID: https://orcid.org/0000-0002-2941-6953 Centro Universitário Tiradentes, Brasil

E-mail: rafaela.andrade84@ souunit.com.br

Dannyele Cynthia Santos Pimentel Nicácio

ORCID: https://orcid.org/0000-0002-2664-1688 Centro Universitário Tiradentes, Brasil E-mail: danny_cynthia@hotmail.com

\section{Resumo}

A avulsão dentária consiste em um trauma dentoalveolar, no qual ocorre o deslocamento do dente para fora do alvéolo, sendo o tratamento imediato o reimplante do elemento dental avulsionado. Fatores como: tempo extra alveolar, meio de armazenamento, e a contenção realizada após o reimplante dentário, são determinantes no prognóstico favorável do caso. O objetivo desse artigo foi relatar o caso clínico de avulsão do incisivo central superior. Paciente do gênero masculino, 7 anos de idade, sofreu queda da própria altura, ocasionando a avulsão do elemento dental 11; quatro horas após o acidente, com o elemento tendo sido armazenado em meio seco, o reimplante foi realizado e confeccionada uma contenção rígida (barra de Erich). Em atendimento posterior, o elemento em questão foi avaliado e a contenção substituída por uma semi-rígida; reavaliado e encaminhando para tratamento endodôntico. O caso relatado trata-se de um caso atípico, para o quadro clínico em questão os fatores citados foram desfavoráveis para um bom prognóstico, entretanto, o elemento que sofreu avulsão e foi reimplantado, encontra-se em função até o presente momento, com 9 meses de acompanhamento.

Palavras-chave: Traumatismo dentoalveolar; Avulsão dentária; Reimplante dentário.

\begin{abstract}
Dental avulsion consists of a dentoalveolar trauma, in which the tooth is displaced out of the alveolo, and immediate treatment is the reimplantation of the avulsional dental element. Factors such as: extra alveolar time, storage medium, and the containment performed after dental reimplantation, are determinant in the favorable prognosis of the case. The aim of this article was to report the clinical case of avulsion of the upper central incisor. A 7-year-old male patient suffered a fall from his own height, causing avulsion of the dental element 11; four hours after the accident, with the element having been stored in dry medium, the reimplantation was performed and a rigid containment was made (Erich bar). In later care, the element in question was evaluated and the containment replaced by a semi-rigid one; reevaluated and referring for endodontic treatment. The case reported is an atypical case, for the clinical picture in question the factors mentioned were unfavorable for a good prognosis, however, the element that suffered avulsion and was reimplanted, is in function until the present moment, with 9 months of follow-up.
\end{abstract}

Keywords: Dentoalveolar trauma; Dental avulsion; Dental reimplantation.

\section{Resumen}

La avulsión dental consiste en un trauma dentoalveolar, en el cual el diente se desplaza fuera del alveolo, y el tratamiento inmediato es el reimplantation del elemento dental avulsional. Factores como: el tiempo extra alveolar, el 
medio de almacenamiento y la contención realizada después del reimplantamiento dental, son determinantes en el pronóstico favorable del caso. La puntería de este artículo era divulgar el caso clínico de la avulsión del incisivo central superior. Un paciente masculino de 7 años sufrió una caída de su propia altura, causando la avulsión del elemento dental 11; cuatro horas después del accidente, con el elemento almacenado en medio seco, se realizó la reimplantación y se realizó una contención rígida (barra de Erich). En cuidados posteriores, se evaluó el elemento en cuestión y se sustituyó la contención por uno semirrígido; reevaluado y refiriendo para el tratamiento endodontic. El caso relatado es un caso atípico, para el cuadro clínico en cuestión los factores mencionados fueron desfavorables para un buen pronóstico, sin embargo, el elemento que sufrió avulsión y fue reimplantado, está en función hasta el momento presente, con 9 meses de seguimiento.

Palabras clave: Traumatismo dentoalveolar; Avulsión dental; Reimplantación dental.

\section{Introdução}

O traumatismo dentário é um dano causado ao dente e/ou estruturas de suporte do mesmo. A etiologia pode variar desde acidentes domésticos, ciclísticos, automobilísticos, atividades esportivas e ocasionando diversos tipos de traumas dentários. A depender do impacto, pode comprometer a estética, gerando problemas psicológicos e sociais para o indivíduo (Costa et al., 2014). O trauma dentário pode englobar desde uma pequena fratura do esmalte até a perda definitiva do elemento dentário. Alterações como perda de estrutura dentária, sensibilidade, presença de dor, mobilidade dentária, reabsorções radiculares e necrose pulpar, podem ser observadas nos indivíduos que sofreram trauma dentários (Sanabe et al., 2009). Em relação a prevalência dos traumas dentários, os grupos mais acometidos são os de crianças e adolescentes, atingindo a dentição permanente é de 8 até 12 anos. Dentre os elementos dentais o mais suscetível é o incisivo central superior, que engloba $80 \%$ das injúrias dentárias, seguido pelo lateral superior e aos incisivos centrais e laterais inferiores (Lopes \& Siqueira et al., 2020).

A classificação desses traumas, dar-se pela região que sofreu a injúria, bem como sua extensão, podendo acometer desde a coroa até a raiz, sendo descritas como: fraturas coronárias, fraturas coroa-raiz, fraturas radiculares, injúrias por luxações, sendo a mesma classificadas em: concussão, subluxação, luxação lateral, luxação extrusiva, luxação intrusiva e avulsão (Lopes \& Siqueira et al., 2020).

A avulsão dentária, consiste em uma lesão traumática complexa no tecido periodontal e pulpar, uma vez que nesse tipo de trauma o dente é completamente deslocado da cavidade alveolar (Hicks et al., 2016). Geralmente envolve os dentes centrais superiores, geralmente em indivíduos que apresentam selamento labial inadequado e que possuem protrusão da maxila maior que $5 \mathrm{~mm}$ em relação à mandíbula são mais suscetíveis à ocorrência de traumatismos dentário que trazem uma grande harmonia para a estética do sorriso (Soriano et al., 2004). Podendo ainda prejudicar o desenvolvimento ósseo alveolar em crianças que não completaram a formação óssea. (Demir et al., 2020).

O reimplante dentário é o tratamento de escolha para os casos de avulsão dentária, entretanto, o prognóstico é variado, podendo ser influenciado por fatores clínicos e demográficos, relacionados ao manejo do dente avulsionado, ao tratamento e ao perfil de resposta do hospedeiro (Amaro et al., 2020). Dentre esses fatores, pode-se destacar o período de tempo extraalveolar, e nos casos em que o reimplante não foi realizado imediatamente, o local de armazenamento adequado, além da contenção realizada pós reimplante.

Em relação ao tempo extra alveolar, quanto menor for sua permanência fora do alvéolo, mais favorável será o prognóstico. Um período de tempo considerado ideal é entre 15 a 20 minutos para que possa haver reparação periodontal, após o reimplante (Lopes \& Siqueira et al., 2020). Um outro fator determinante é o meio de armazenamento, até a realização do reimplante, o elemento dental pode ser preservado por um curto período em meio apropriado, como leite, soro fisiológico, saliva ou solução de Hank, no entanto, isso nem sempre é possível (Demir et al., 2020). Após o reimplante, um fator também importante é a confecção de uma contenção apropriada, para permitir a alocação e cicatrização do dente no alvéolo, o tipo de contenção deve ser a contenção semiflexível ou semirrígida (Hupp et al., 2021).

Diante do exposto, o reimplante dentário após trauma de avulsão tem sido conceituado como uma conduta de 
tratamento positiva de primeira escolha segundo a literatura, tendo como propósito a preservação da estrutura do elemento dental e estética do sorriso. Assim sendo, o objetivo do presente trabalho é relatar um reimplante após trauma dentário de avulsão no incisivo central superior

\section{Metodologia}

Este artigo trata-se de um relato de caso, abordado de forma descritiva e qualitativa, onde segundo Pereira et al. (2018), caracteriza-se como uma pesquisa que por via direta recolhe dados relativos ao estudo através do acesso aos registros e aos exames fornecidos, sendo o pesquisador o instrumento primordial. O presente estudo trata-se de um relato de caso clínico com tratamento de reimplante dentário em paciente odontopediátrico. Todas as informações em questões foram colhidas através do prontuário do paciente, além de exames de imagem com o intuito de descrever o caso. No que se relacionam aos aspectos éticos, esclarecimentos sobre riscos, benefícios e prognósticos foram fornecidos ao paciente por meio do Termo de Consentimento Livre e Esclarecido (TCLE) e a autorização do procedimento ocorreu mediante assinatura de tal documento.

\section{Relato de Caso Clínico}

Paciente 7 anos de idade, do sexo masculino, procurou atendimento no Hospital Geral do Estado de Alagoas (HGE) no dia 17 de setembro de 2020. A criança sofreu uma queda da própria altura transcorrendo em um traumatismo dentário (avulsão) no incisivo central superior (elemento dental 11). O dente foi armazenado em um recipiente de plástico descartável sem líquido e envolvido em papel toalha seco.

Devido ao paciente ser do interior, após 4 horas do incidente, o paciente chegou ao serviço hospitalar e ao ser atendido no hospital, a equipe plantonista realizou o reimplante do elemento, em seguida foi realizado a contenção rígida com barra de erich e fio de aço. Realizou-se a prescrição de $45 \mathrm{mg} / \mathrm{kg} / \mathrm{dia}$ amoxicilina por 7 dias e $200 \mathrm{mg}$ de ibuprofeno por 4 dias, junto com o bochecho com clorexidina $0,12 \%$.

Ao ser atendido na clínica escola de Odontologia do Centro Universitário Tiradentes (UNIT-AL) 12 dias após o trauma no dia 29 de setembro de 2020, foi realizada a radiografia inicial (Figura 1) em seguida remoção de sua contenção da barra de erich (Figura 2), feita nova contenção com fio ortodôntico 0,8mm (Figura 3) e solicitado exame de tomografia computadorizada para avaliar o elemento 11. 
Research, Society and Development, v. 10, n. 8, e4910816967, 2021

(CC BY 4.0) | ISSN 2525-3409 | DOI: http://dx.doi.org/10.33448/rsd-v10i8.16967

Figura 1: Radiografia inicial (29 de setembro de 2020).

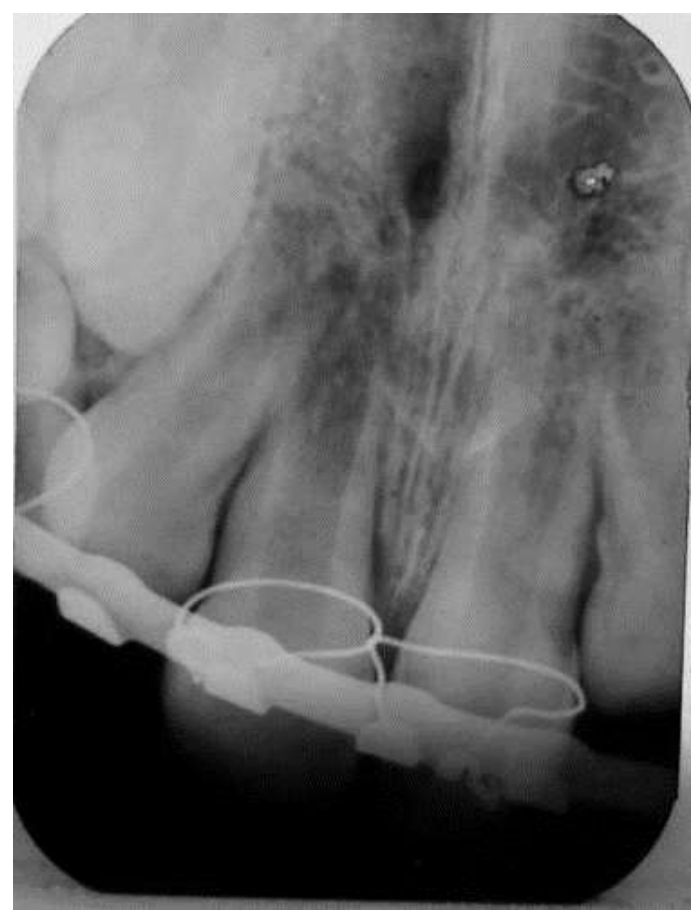

Fonte: Autores.

Figura 2: Contenção com a barra de erich (29 de setembro de 2020).

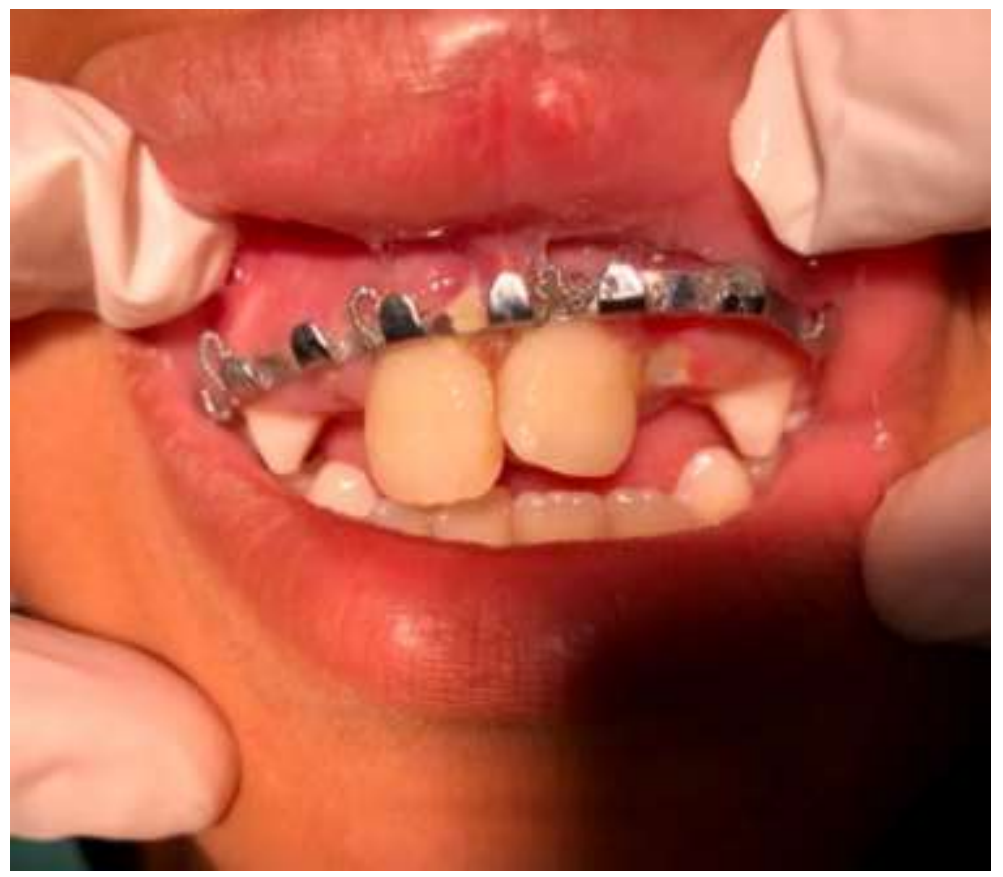

Fonte: Autores. 
Figura 3: contenção com fio ortodôntico 0,8mm (29 de setembro de 2020).

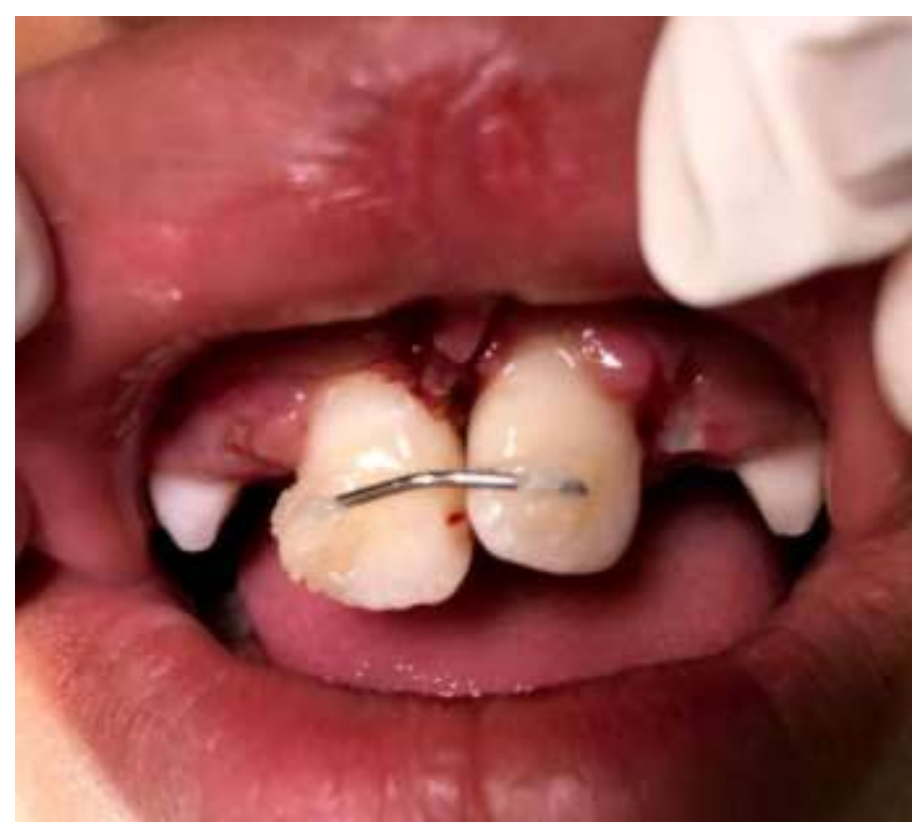

Fonte: Autores.

Em seguida após 8 dias foi realizada a avaliação do incisivo central superior (elemento dental 11), foi feito o teste de sensibilidade dentária com o frio o qual apresentou o resultado negativo para o teste de sensibilidade, da mesma forma o protocolo nos dentes vizinhos (elementos dentários 21 e 12) para avaliar possíveis traumas devido ao acidente. Ainda nesta consulta observou-se ausência de edema, sem alteração de tecidos moles. Não foi feita a remoção da contenção com fio ortodôntico pela mobilidade do dente e isso acarretou pela permanência dela.

O paciente retornou para avaliação e análise da tomografia computadorizada (Figura 4-7) não foi observada fratura do incisivo central superior (elemento dental 11). Realizou-se a remoção da contenção do fio ortodôntico (Figura 8) na clínica escola de Odontologia do Centro Universitário Tiradentes (UNIT-AL), no dia 21 de janeiro de 2021 e posteriormente o paciente foi encaminhado para realizar tratamento endodôntico. 
Figura 4: Tomografia 3D.

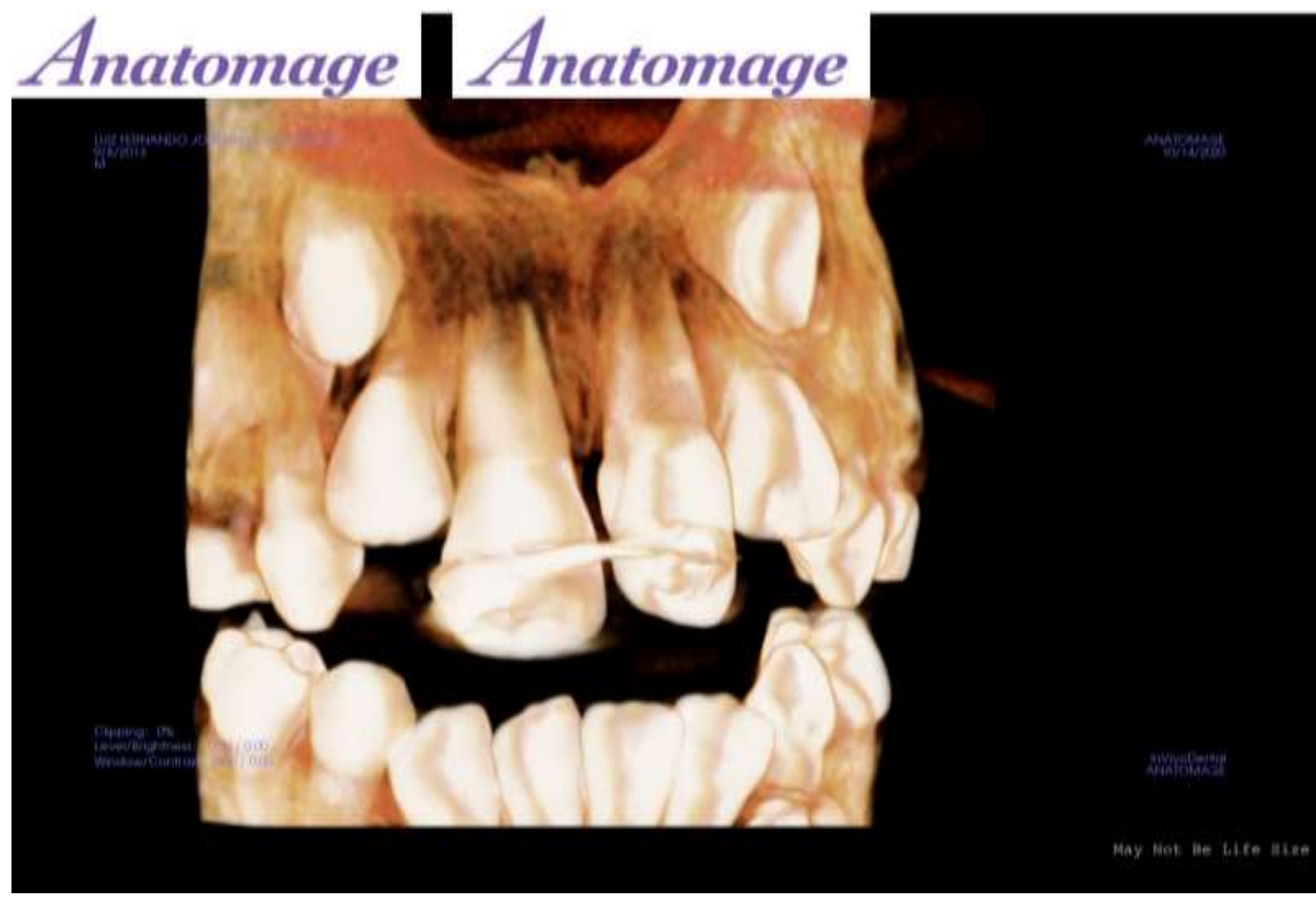

Fonte: Autores.

Figura 5: Cortes axiais, para permitir obter uma imagem nítida de um só plano de corte da maxila.

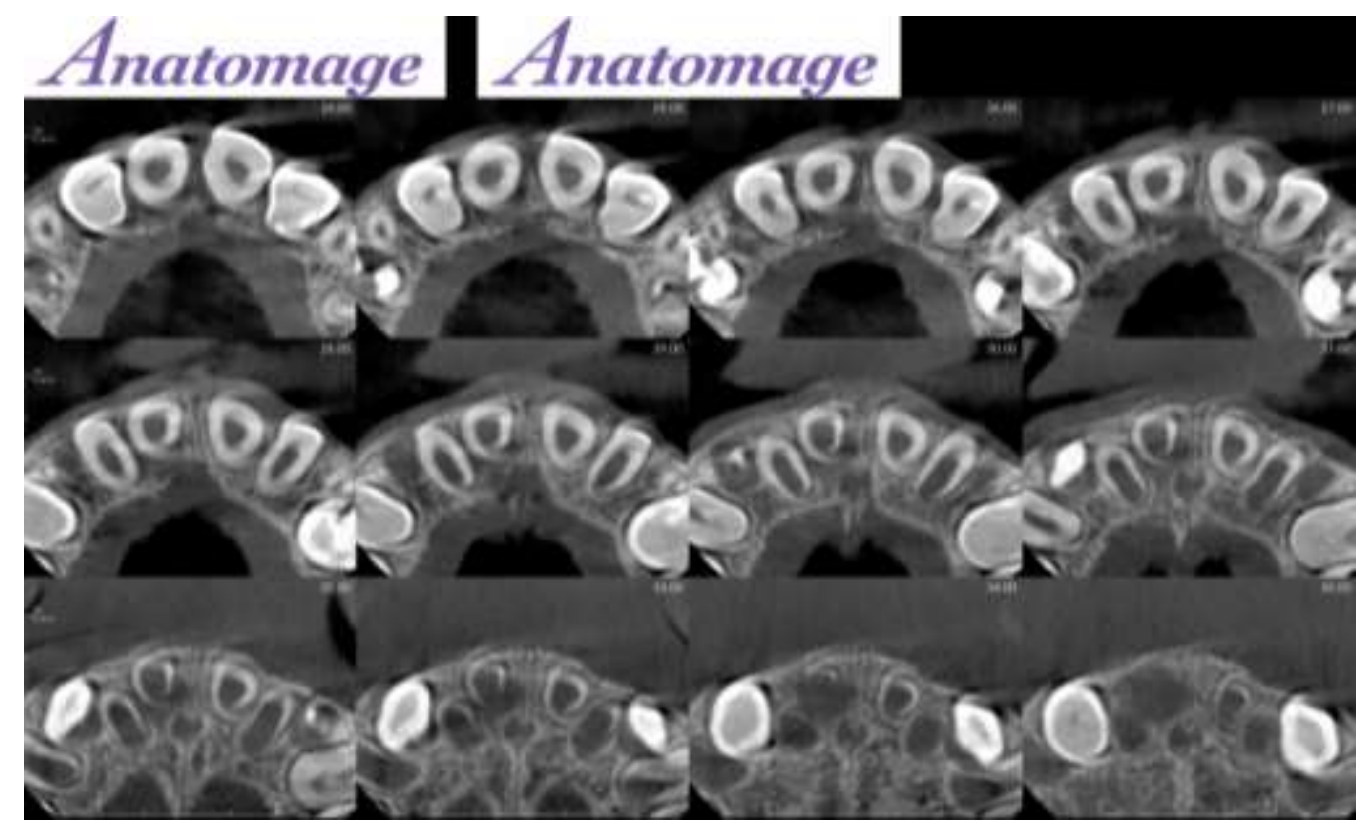

Fonte: Autores. 
Figura 6: Corte anterior da maxila, para melhor vizualização da região anterior e analizar que não há fratura radicular no elemento 11 .

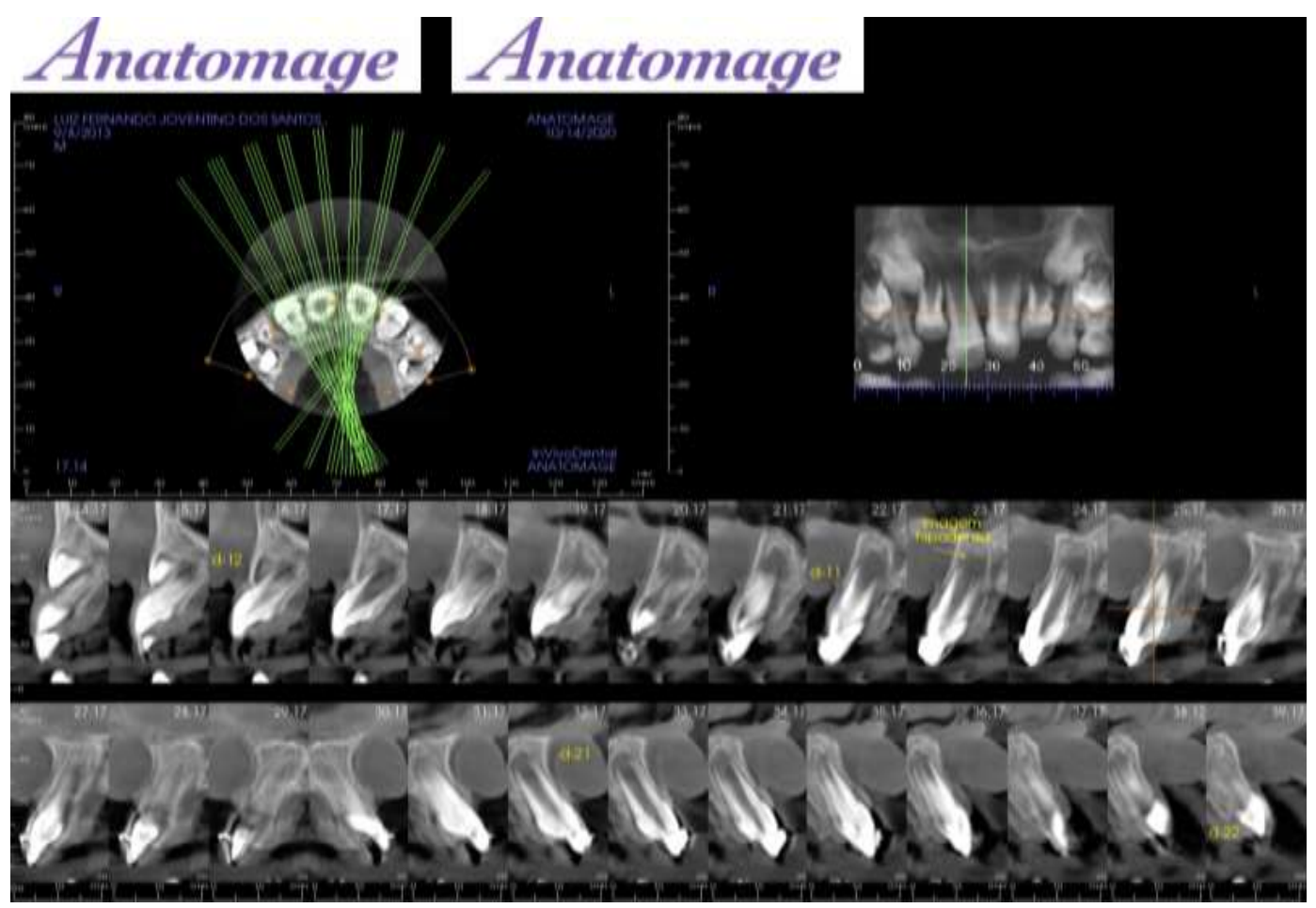

Fonte: Autores.

Figura 7: Cortes coronais, não há fratura radicular no elemento 11.

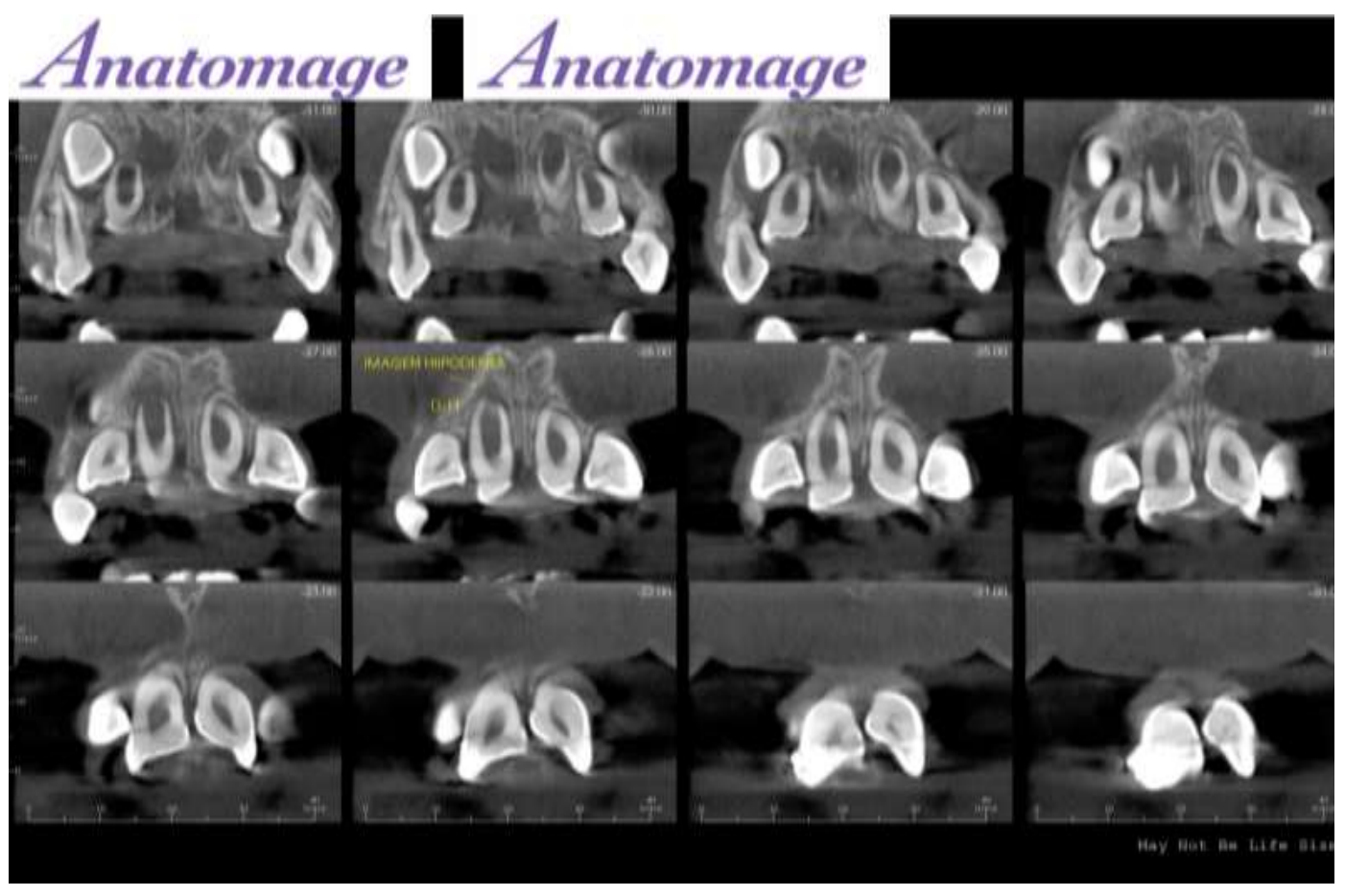

Fonte: Autores. 
Figura 8: elemento sem contenção (21 de janeiro de 2021)

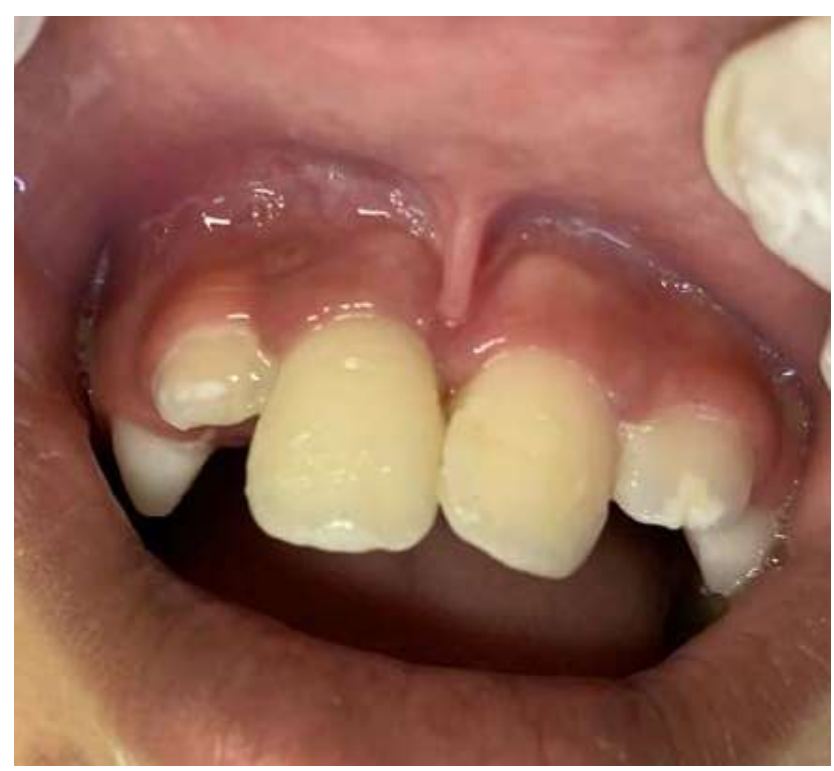

Fonte: Autores.

\section{Discussão}

Os traumatismos dento-alveolares são habituais, porém devem ser priorizados como caso emergencial, pois o sucesso depende do atendimento prestado no momento em que ocorreu o trauma (Hupp et al., 2021). Sabe-se que esses tipos de traumas dento-alveolares são um problema de saúde pública, não por serem comuns, mas por afetar a vida do paciente, com considerações estéticas, funcionais e psicológicas causando algum desconforto. É notório o quanto o paciente é afetado, tanto fisicamente, quanto psicologicamente, após um traumatismo dentário, principalmente quando há envolvimento dos elementos dentários anteriores (Trabert et al., 2003; Oliveira et al., 2004 e Moraes et al., 2011). Apesar do paciente do caso clínico em questão ser uma criança, o mesmo relatou que estava com medo de não poder sorrir novamente, após avulsão do elemento 11.

A prevalência de acometimento de traumas em incisivo central superior permanente é de 80\% dos casos (Lopes \& Siqueira et al., 2020). Em concordância, Fernandes et al., (2005) e Miloro, (2016) relatam que os casos de as avulsões geralmente acometem um único dente, sendo o incisivo central superior o de maior prevalência. E ainda que esse tipo de trauma atinge, na maioria dos casos, crianças de 7 a 9 anos, ocorrendo com maior frequência na dentição permanente. Corroborando com as prevalências citadas, no presente estudo, ocorreu o trauma de avulsão em uma criança de 7 anos de idade, envolvendo o incisivo central superior permanente (o elemento dental 11).

Nos casos de traumas dentários, por avulsão, o tratamento de escolha é o reimplante dentário. Hupp et al., (2021) recomendam que ao ocorrer avulsão total de um dente, os responsáveis devem lavar o dente imediatamente com a saliva do paciente, água filtrada ou soro fisiológico e realize o reimplante imediato. E ainda enfatiza que o manejo deve ser sempre pela coroa dentária, tentando não tocar na raiz, reposicioná-lo e procurar imediatamente o cirurgião-dentista.

O sucesso do reimplante dentário, depende de alguns fatores, tais como: tempo fora do alvéolo e o meio de conservação, nos casos em que não é possível realizar o reimplante imediato, seja por falta de conhecimento ou por inconsciência pós-trauma do paciente (Andreasen, 1995; Granville-Garcia, 2007; Curylofo et al., 2012). Deve-se considerar também, um outro fator, a realização de uma contenção adequada após o reimplante (Hupp et al., 2021). Em relação ao tempo extra alveolar, sabe-se que quanto menor o tempo fora do alvéolo, melhor será o prognóstico do caso, segundo Stokes e colaboradores (1992) o reimplante realizado em até 30 minutos apresenta alta taxa de sucesso, ainda segundo Lopes \& Siqueira et al., (2020), um período de tempo considerado ideal é entre 15 a 20 minutos para que possa haver reparação periodontal. 
Entretanto, Miloro e colaboradores (2016) relatam que essa taxa de sucesso é possível em tempo maior, nas primeiras duas horas. Em discordância Soares e Soares (1998), relata que períodos extra-alveolares superiores a 2 (duas) horas quase sempre determinam extensas reabsorções radiculares, além disso, após 30 minutos fora do alvéolo, dar-se início a um processo degeneração das células do ligamento, consequentemente o percentual de sucesso diminui, bem como o prognóstico, que se torna desfavorável. No presente caso clínico o reimplante ocorreu, 4 horas após o trauma de avulsão, divergindo dos períodos de tempo descritos na literatura, que relatam maiores taxas de sucesso.

Andreasen e Andreasen (2001) trazem meios de conservação, para dentes avulsionados, que permitem a cicatrização dos tecidos pulpar e periodontal, que são solução salina fisiológica, sangue, meios de cultura de tecido e saliva. Poi et al., (2013); Adnan et al., (2018) e Albuquerque et al., (2014) relatam que além desses, há clara de ovo, própolis, e água como meios de armazenamento de dente avulsionado. Miloro et al., (2016) acrescentam que a solução de Hanks como meio compatível fisiologicamente.

Hupp et al., (2021), Silva Júnior et al., (2015) e Reis (2013) convergem ideias e relatam que o meio de armazenamento de primeira escolha é a solução salina de Hanks, por preservar os metabólitos e propor vitalidade às células do ligamento periodontal, por ser compatível com a osmolaridade e o pH adequado do elemento dental. Por ter composição de nutrientes essenciais, por exemplo o cálcio, glicose e íons de magnésio que conservarão a vitalidade das células. Sendo a solução de Hanks importante para reduzir a incidência de anquilose por melhorar a sobrevivência das células periodontais da superfície radicular, também colabora com a remoção dos resíduos da raiz e dissolve as bactérias. No entanto, vale ressaltar essa solução não é facilmente comercializada.

Um meio de armazenamento mais facilmente acessível e conhecido pela população, em geral, é o leite, tido como um meio de conservação de até 6 (seis) horas, sendo de boa disponibilidade e eficaz, pelo Ph básico, segundo Patil \& Sydiskis, (1994). Em contrapartida, os autores Sardana, Goyal e Gauba (2014) conseguiram um bom prognóstico após avulsão, como mostrado em seu caso, o qual conservou o dente por 15 horas, submergido ao leite. Já Hiltz (1991) e Blomlof et al., (1983) relatam que o leite é um ótimo meio de armazenamento quando se trata de avulsão devido a sua osmolaridade e pH, além da disponibilidade e que conserva por até 8 horas. O caso clínico descrito, como já mencionado, pelas circunstâncias locais, se diferencia na forma de armazenamento e no tempo extra-alveolar, por não ser favorável, já que foi em meio seco (papeltoalha), e o tempo de 4 horas do local do trauma até o exato momento do reimplante, um dos fatores que implica o sucesso segundo Andreasen et al., (1995), visto que houve um grande intervalo entre o trauma e o reimplante. O autor cita que em meio seco, não passar de 1 (uma) hora para reimplante, é um fator importante no sucesso do tratamento. Tanto Miloro et al., (2016), quanto Andreasen e colaboradores (1995) destacam que o tempo excedido para realização do reimplante pode ser causa de reabsorção óssea, anquilose e insucesso do reimplante, principalmente quando em meio seco. A AOCMF (2012) ressalta que, nos casos de avulsão dentária, em meio seco ultrapassando 30 (trinta) minutos para tratamento, deixando baixa a chance de sucesso. No entanto, o caso clínico em questão, apesar de exceder o tempo em meio seco por 4 (quatro) horas, houve sucesso clínico e radiográfico (fig. 4), sem reabsorção e com sinais de sendo este caso um desvio padrão da literatura. E que até o presente momento, encontra-se em 8 (oito) meses de follow-up.

Hupp e colaboradores (2021), declaram que após o reimplante dentário, deve-se ser confeccionada uma contenção, para permitir a alocação e cicatrização do dente no alvéolo, o tipo de contenção deve ser a contenção semiflexível ou semirrígida, idealmente aquelas que facilitem higienização, remoção e não prejudiquem o tecido periodontal. Miloro et al., (2016) traz algumas alternativas como compósitos resinosos, podendo associar fio de nylon, assim como o de aço, fino, que segundo a International Association of Dental Traumatology IADT (2012) é recomendado o de 0,4 mm. Segundo Hupp et al., (2021) até um clip de papel pode ser associado à resina composta para estabilização.

Schein e Isolan (1997); Von Arx, Fillippi, Buser (2001); Antrim e Ostrowski (1982) sugerem que seja feita a 
esplintagem semirrígida com fio de nylon e resina composta durante um período de uma a duas semanas. Já o Miloro et al., (2016) recomendam de 7 a 10 dias. Andreasen e colaboradores (1992) sugerem a retirada da contenção após 7 dias, para permitir certo movimento funcional do dente reimplantado e reduzir o risco de anquilose. Com isso, a esplintagem semirrígida favorecerá a cicatrização pulpar e periodontal, além de diminuir a incidência de anquilose e reabsorção por substituição bem como a estabilização dentária durante o período de cicatrização. Apesar disso, ocorreu sucesso (até o presente momento) com o caso clínico especificado aqui, mesmo com a contenção com a barra de erich, feita inicialmente, no serviço de emergência, se tratando de uma contenção rígida, não sendo indicada pela IADT (2020) e AOCMF (2012), bem como pela literatura, em geral.

Segundo AOCMF (2012) a barra de Erich é um dispositivo utilizado para fixação maxilomandibular, sendo uma contenção rígida. Ferreira et al (2004) ainda mencionam suas indicações no tratamento fechado de fraturas de mandíbula em região de sínfise, corpo, ângulo, em fraturas verticais de ramo, colo de côndilo, fraturas alveolares de maxila ou mandíbula e ancoragem de dentes avulsionados. Miloro et al., (2016), inclusive, destacam que a barra de erich para esse tipo de procedimento não é mais recomendado. Apesar de Hupp et al., (2021) ainda mencionar as barras e fios de aço como uma possibilidade de tratamento. No caso clínico relatado o paciente ficou com a contenção com a barra de erich durante 12 dias podendo acometer a vitalidade do seu periodonto, divergindo da literatura que mesmo assim, não obteve agravos significativos com a contenção e sua vitalidade periodontal. A contenção semirrígida não foi realizada, inicialmente, no presente trabalho devido ao local de pronto atendimento não possuir resina composta e materiais para confecção, sendo necessário utilizar o que possuía no local que era fio de aço e barra de erich.

Hupp et al., (2021) garante que durante o período de cicatrização, a inflamação deve ser reduzida, para não causar a reabsorção inflamatória da raiz, o que corrobora a desvantagens da imobilização por fio de aço e dos esplintes de resina acrílica. Além de possuir uma dificuldade em limpar os dentes com fios de aço ou esplintes. Outrossim, o fio de aço tem a possibilidade de deslizar apicalmente ao redor da margem cervical e lesionar o cemento. No caso clínico exposto foi realizado em sua segunda sessão a contenção semirigida com a confecção de nova contenção com fio ortodôntico $0,8 \mathrm{~mm}$ e resina composta, devido a mobilidade existente no dente do paciente. Pôde-se observar que é um caso atípico, pois não teve surgimento de anquilose e não foi reabsorvido até o presente momento desde o reimplante, até o $9^{\circ}$ mês após o trauma de avulsão.

\section{Considerações Finais}

O sucesso do tratamento de reimplante dentário, após trauma de avulsão é essencial para devolver a estética, função, autoestima e harmonia do paciente. Os procedimentos realizados no paciente neste caso em particular foram satisfatórios pós a realização dos atendimentos, mesmo sendo um caso atípico, divergindo com as descrições da literatura, tanto em relação ao tempo extra alveolar (4 horas) quanto ao meio de armazenamento (papel seco), e ainda temporariamente com uso de uma contenção rígida. O mesmo demonstra uma evolução inesperada, porém, significativa, já que até o presente momento (proservação de 9 meses) o elemento dental encontra-se em boca e em função.

\section{Referências}

Adnan, S., Lone, M. M., Khan, F. R., Hussain, S. M., \& Nagi, S. E. (2018). Which is the most recommended medium for the storage and transport of avulsed teeth? A systematic review. Dental Traumatology, 34(2), 59-70.

Albuquerque, Y. E., Rosell, F. L., da Silva Tagliaferro, E. P., \& da Silva, S. R. C. (2014). Conhecimento de mães sobre os procedimentos de emergência nos casos de avulsão dentária. Revista da Faculdade de Odontologia-UPF, 19(2).

Amaro, R. G. (2020). Prognóstico pulpar de dentes permanentes com rizogênese incompleta reimplantados após avulsão traumática: estudo clínico longitudinal. Dissertações de Mestrado, Universidade Federal de Minas Gerais, Belo Horizonte, MG, Brasil. 
Andersson, L., Andreasen, J. O., Day, P., Heithersay, G., Trope, M., DiAngelis, A. J., ... \& Tsukiboshi, M. (2012). International Association of Dental Traumatology guidelines for the management of traumatic dental injuries: 2. Avulsion of permanent teeth. Dental Traumatology, 28(2), 88-96.

Andreasen, J. O., \& Andreasen, F. M. (2001). Fundamentos de traumatismo dental: guia de tratamento passo a passso. Artmed.

Andreasen, J. O., Borum, M. K., Jacobsen, H. L., \& Andreasen, F. M. (1995). Replantation of 400 avulsed permanent incisors. 4. Factors related to periodontal ligament healing. Dental traumatology, 11(2), 76-89.

Andreasen, J. O. (1992). Third International Conference on Dental Trauma. Dental Traumatology, 8(2), 67-68.

Antrim, D. D., \& Ostrowski, J. S. (1982). A functional splint for traumatized teeth. Journal of endodontics, 8(7), 328-331.

Bourguignon, C., Cohenca, N., Lauridsen, E., Flores, M. T., O'Connell, A. C., Day, P. F., ... \& Levin, L. (2020). International Association of Dental Traumatology guidelines for the management of traumatic dental injuries: 1. Fractures and luxations. Dental Traumatology, 36(4), 314-330.

Blomlöf, L., Lindskog, S., Andersson, L., Hedström, K. G., \& Hammarström, L. (1983). Storage of experimentally avulsed teeth in milk prior to replantation. Journal of Dental Research, 62(8), 912-916.

Costa, L. E. D., Queiroz, F. D. S., Nóbrega, C. B. C., Leite, M. S., Nóbrega, W. F. S., \& Almeida, E. R. D. (2014). Trauma dentário na infância: avaliação da conduta dos educadores de creches públicas de Patos-PB. Revista de Odontologia da UNESP, 43(6), 402-408.

Curylofo, P. A., Lorencetti, K. T., \& da Silva, S. R. C. (2012). Avaliação do conhecimento de professores sobre avulsão dentária. Arquivos em Odontologia, 48(3).

Demir, P. I. N. A. R., Guler, C., Kizilci, E., \& Keskin, G. (2020). Survival of avulsed permanent incisors in children following delayed replantation. Nigerian journal of clinical practice, 23(5), 631-637.

Prein J., Ehrenfeld, M., Manson P. N., (2012). Principles of Internal Fixation of the Craniomaxillofacial Skeleton - Trauma and Orthognathic Surgery. AO CMF manuals. Thieme New York, (103-107)

Fernandes, J. L., Viana, S. L., Mendonca, J. L. F. D., Freitas, F. M. O., Lima, G. A. D. S., Vila, A. F. D., \& Ribeiro, N. (2005). Avulsion fractures of the anterior inferior iliac spine: spectrum of imaging findings; Avulsao da espinha iliaca antero-inferior: espectro dos achados de imagem. Revista da Imagem, 27.

Ferreira, A. G. M., Weismann, R., Heitz, C., de Oliveira, M. G., \& Woitchunas, G. F. P. (2004). Três métodos de bloqueio maxilomandibular para o tratamento das fraturas mandibulares. Revista da Faculdade de Odontologia-UPF, 9(2).

Granville-Garcia, A. F., Lima, E. M., Santos, P. G., \& de MENEZES, V. A. (2007). Avaliação do conhecimento dos professores de educação física de Caruaru-PE sobre avulsão-reimplante. Pesquisa Brasileira em Odontopediatria e Clínica Integrada, 7(1), 15-20.

Hicks, R. W., Green, R., \& Van Wicklin, S. A. (2016). Dental avulsions: Review and recommendations. The Nurse Practitioner, 41(6), 58-62.

Hiltz, J., \& Trope, M. (1991). Vitality of human lip fibroblasts in milk, Hanks balanced salt solution and Viaspan storage media. Dental Traumatology, 7(2), 69-72.

Hupp, J. R., Ellis, I., \& Tucker, M. R. (2021). Cirugia oral e maxilofacial contemporanea. Rio de Janeiro Rio de Janeiro: GEN Grupo Editorial Nacional S.A. Editora Guanabara Koogan Ltda. (7a ed.), (506-513).

Lopes, H. P.; Siqueira Júnior, J.F (2020). Endodontia: biologia e técnica. (5a ed.), Guanabara Koogan.

Miloro, M. Ghali, G. E., Larsen, P. E., Waite, P. D., (2016). Princípios de cirurgia bucomaxilofacial de Peterson. In Princípios de cirurgia bucomaxilofacial de Peterson (3a ed.), (pp. 324-324).

Moraes, R. S. D., Silva, R. V. D., Félix Junior, W. D. S., Costa, D. J. D., \& Rebellato, N. L. B. (2011). Tratamento emergencial de traumatismo dentoalveolar associado a dente decíduo fusionado: relato de caso. Revista de Cirurgia e Traumatologia Buco-maxilo-facial, 11(3), 39-42.

Oliveira, F. A. M., Gerhardt de Oliveira, M., Orso, V. A., \& Oliveira, V. R. (2004). Traumatismo dentoalveolar: revisão de literatura dentoalveolar traumatism: literature review. Revista de Cirurgia e Traumatologia Buco-maxilo-facial, 4(1), 15-21.

Patil, S., DUMSHA*, T. C., \& Sydiskis, R. J. (1994). Determining periodontal ligament (PDL) cell vitality from exarticulated teeth stored in saline or milk using fluorescein diacetate. International endodontic journal, 27(1), 1-5.

Poi, W. R., Sonoda, C. K., Martins, C. M., Melo, M. E., Pellizzer, E. P., Mendonça, M. R. D., \& Panzarini, S. R. (2013). Storage media for avulsed teeth: a literature review. Brazilian Dental Journal, 24(5), 437-445.

Reis, M. V. D. P. (2013). Avaliação de diferentes meios de armazenagem para dentes avulsionados: estudo experimental em cães. Dissertação, Universidade Federal de Uberlândia, Uberlândia, MG, Brasil.

Sanabe, M. E., Cavalcante, L. B., Coldebella, C. R., \& Abreu-e-Lima, F. C. B. D. (2009). Urgências em traumatismos dentários: classificação, características e procedimentos. Revista Paulista de Pediatria, 27(4), 447-451.

Sardana, D., Goyal, A., \& Gauba, K. (2014). Delayed replantation of avulsed tooth with 15-hours extra-oral time: 3-year follow-up. Singapore dental journal, $35,71-76$.

Schein, M. T., \& Isolan, T. M. P. (1997). Esplinte funcional de dentes traumatizados. Revista brasileira de odontologia, 225-7. 
Research, Society and Development, v. 10, n. 8, e4910816967, 2021

(CC BY 4.0) | ISSN 2525-3409 | DOI: http://dx.doi.org/10.33448/rsd-v10i8.16967

Silva Júnior, E. Z. D., Silva, T. M. V. D., Esteves, G. B., Rolim, H. S. F., \& Dourado, A. C. A. G. (2015). Prognóstico e tratamento da avulsão dentária: relato de caso. Revista de Cirurgia e Traumatologia Buco-maxilo-facial, 15(3), 39-42.

Soares, I. M. L., \& Soares, I. J. (1988). Técnica do reimplante dental: tratamento dos dentes traumatizados e conduta clínica para reimplantação. RGO. Revista Gaúcha de Odontologia (Porto Alegre), 331-6.

Soriano, E. P., Caldas Jr, A. F., \& Góes, P. S. A. (2004). Risk factors related to traumatic dental injuries in Brazilian schoolchildren. Dental traumatology, 20(5), 246-250.

Stokes, A. N., Anderson, H. K., \& Cowan, T. M. (1992). Lay and professional knowledge of methods for emergency management of avulsed teeth. Dental Traumatology, 8(4), 160-162.

Trabert, J., Perez, M. A., Blank, V., Bocil, R. S., \& Pietnuza, J. A. (2003). Prevalence of traumatic dental injuries and associate factors among 12 years-old schoolchildren in Florinopolis. Brazil. Dent Traumatol, 19, 15-18.

Von Arx, T., Filippi, A., \& Buser, D. (2001). Splinting of traumatized teeth with a new device: TTS (Titanium Trauma Splint). Dental Traumatology, 17(4), 180-184. 